\title{
Explaining Gentzen's Consistency Proof within Infinitary Proof Theory
}

\author{
Wilfried Buchholz \\ Mathematisches Institut \\ der Ludwig-Maximilians-Universität München \\ Theresienstr. 39, D-80333 München, Germany \\ email: buchholz@rz.mathematik.uni-muenchen.de
}

\section{Introduction}

There are two main approaches to ordinal analysis of formal theories: the finitary Gentzen-Takeuti approach on one side, and the use of infinitary derivations initiated by Schütte on the other. Up to now these approaches where thought of as separated and only vaguely related. But in the present paper we will show that actually they are intrinsically connected. Using the concept of notations for infinitary derivations (introduced in [Bu91]) a precise explanation of Gentzen's reduction steps on derivations in 1st order arithmetic $Z$ (cf. [Ge38]) in terms of (cut-elimination for) infinitary derivations in $\omega$-arithmetic will be given. Even more, Gentzen's reduction steps and ordinal assignment will be derived from infinitary proof theory. In a forthcoming paper we will extend the present work to impredicative subsystems of 2nd order arithmetic thereby explaining Takeuti's consistency proof for $\Pi_{1}^{1}$-CA in terms of the infinitary approach (with $\Omega_{\mu+1}$-rules) from [BS88] (cf. [Bu97]).

Our general idea is that such investigations may perhaps be helpful for the understanding and unification of two of the most advanced achievements in contemporary proof theory, namely the methodically quite different work of T. Arai ([Ar96b], [Ar97a], [Ar97b]) and M. Rathjen ([Ra91], [Ra94], [Ra95]) on the ordinal analysis of very strong subsystems of 2 nd order arithmetic and set theory.

\section{Content}

In $\S 1$ and $\S 2$ essential material from [Bu91] is repeated in a somewhat modified form, so that it fits exactly for the present purpose. $\S 1$ contains the definition of operators $\mathcal{R}_{C}$ and $\mathcal{E}$ which make up a cut-elimination procedure for $\mathbf{Z}^{\infty}$ (the infinitary Tait-style sequent calculus for $\omega$-arithmetic) due to Schütte [Sch51], Tait [Ta68] and Mints [Mi75]. In $\S 2$ we introduce a finitary Tait-style sequent calculus $Z^{*}$ for pure number theory $Z$ which differs from the usual version only by a certain additional inference rule (E) $\frac{\Gamma}{\Gamma}$ and the fact that cuts $\frac{\Gamma, C \quad \Gamma, \neg C}{\Gamma}$ are labeled by the symbol $\mathrm{R}_{C}$ (instead of $\mathrm{Cut}_{C}$ ). Every $\mathbf{Z}^{*}$-derivation $h$ with closed endsequent is considered as a notation for a certain $\mathbf{Z}^{\infty}$-derivation $h^{\infty}$ of the same sequent. In other words, we define a translation $h \mapsto h^{\infty}$ from $\mathbf{Z}^{*}$ into $\mathbf{Z}^{\infty}$. The definition of $h^{\infty}$ runs as usual only that cuts and E-inferences are not translated literally but according to the intended meaning of the symbols $\mathrm{R}_{C}$, $\mathrm{E}$ : 


$$
\left(\begin{array}{cc}
h_{0} & h_{1} \\
\frac{\Gamma, C}{\Gamma} \Gamma, \neg C \\
\Gamma
\end{array} \mathrm{R}_{C}\right)^{\infty}:=\mathcal{R}_{C}\left(h_{0}^{\infty}, h_{1}^{\infty}\right), \quad\left(\begin{array}{c}
h_{0} \\
\frac{\Gamma}{\Gamma} \mathrm{E}
\end{array}\right)^{\infty}:=\mathcal{E}\left(h_{0}^{\infty}\right) .
$$

From this interpretation and the properties of $\mathcal{R}_{C}$ and $\mathcal{E}$ (established in $\S 1$ ) one immediately reads off a definition of ordinals $\mathrm{o}(h)<\varepsilon_{0}$ and $\operatorname{deg}(h)<\omega$ such that depth $\left(h^{\infty}\right) \leq \mathrm{o}(h)$ and $\sup \left\{\operatorname{rk}(C)+1: C\right.$ is cut-formula in $\left.h^{\infty}\right\} \leq \operatorname{deg}(h)$. Formally the definition of $\mathrm{o}(h)$ and $\operatorname{deg}(h)$ proceeds by (primitive) recursion on the build-up of $h$ and does not refer to $h^{\infty}$. Further by looking on the definitions of $\mathcal{R}_{C}$ and $\mathcal{E}$ (given in $\S 1$ ) we derive (via $h \mapsto h^{\infty}$ ) a definition which assigns to each $\mathbf{Z}^{*}$-derivation $h$ a certain inference $\operatorname{symbol} \operatorname{tp}(h)$ (corresponding to the last inference of $\left.h^{\infty}\right)$ and, for each $i \in|\operatorname{tp}(h)|$, a new $\mathbf{Z}^{*}$-derivation $h[i]$ such that $(h[i])^{\infty}=h^{\infty}(i)$, where $\left(h^{\infty}(i)\right)_{i \in|\operatorname{tp}(h)|}$ is the family of immediate subderivations of $h^{\infty}$. The definition of $\operatorname{tp}(h)$ and $h[i]$ also proceeds by recursion on the build-up of $h$.

In $\S 3$ we describe the (Tait-style adaption of) Gentzen's reduction procedure and ordinal assignment (from [Ge38]) in terms of the notions introduced in $\S 2$. Let $\mathbf{Z}$ denote the subsystem of $\mathbf{Z}^{*}$ obtained by omitting the $\mathbf{E}$-rule. So $\mathbf{Z}$ is just ordinary 1 st order arithmetic. We consider a (hypothetical) Z-derivation $d$ of the empty sequent. Let $d^{\prime}$ be the $\mathbf{Z}^{*}$-derivation which results from $d^{\prime}$ by filling in E-inferences in such a way that for each node $\nu$ of $d^{\prime}$ (which originates from a node of $d)$ we have hgt ${ }^{*}\left(d^{\prime}, \nu\right)=$ Höhe $\left(d^{\prime}, \nu\right)$, where hgt ${ }^{*}\left(d^{\prime}, \nu\right)$ is the number of E's below $\nu$, and Höhe is defined as in [Ge38]. Then o $\left(d^{\prime}\right)$ is precisely the ordinal $O(d)$ which Gentzen assigns to $d$, and $d^{\prime}[0]$ (after deleting all E's) coincides with the result of a Gentzen reduction step applied to $d$.

Remark. The E-rule is also present in [Ar96a] (under the name "height rule") but there no interpretation of $E$ as cut-elimination operator is given.

\section{$\S 1$ Cut-elimination for the infinitary system $z^{\infty}$}

\section{Preliminaries}

We assume a formal language of arithmetic which has predicate symbols for primitive recursive relations, but no function symbols except the constant 0 and the unary function symbol $S$ (successor). Atomic formulas are of the form $p t_{1} \ldots t_{n}$ where $p$ is an $n$-ary predicate symbol and $t_{1}, \ldots, t_{n}$ are terms. Literals are expressions of the shape $A$ or $\neg A$ where $A$ is an atomic formula. Formulas are built up from literals by means of $\wedge, \vee, \forall x, \exists x$. The negation $\neg C$ of a formula $C$ is defined via de Morgan's laws. The $\operatorname{rank} \operatorname{rk}(C)$ of a formula $C$ is defined as usual: $\operatorname{rk}(C):=0$ if $C$ is a literal, $\operatorname{rk}\left(A_{0} \wedge A_{1}\right):=\operatorname{rk}\left(A_{0} \vee A_{1}\right):=\max \left\{\operatorname{rk}\left(A_{0}\right), \operatorname{rk}\left(A_{1}\right)\right\}+1$, $\operatorname{rk}(\forall x A):=\operatorname{rk}(\exists x A):=\operatorname{rk}(A)+1$. By $\operatorname{FV}(\theta)$ we denote the set of all free variables of the formula or term $\theta$. A formula or term $\theta$ is called closed iff $\operatorname{FV}(\theta)=\emptyset$. $\theta_{x}(t)$ (or $\theta(x / t)$ ) denotes the result of replacing every free occurrence of $x$ in $\theta$ by $t$ (renaming bound variables of $\theta$ if necessary). The only closed terms are the 
numerals $0, S 0, S S 0, \ldots$ We identify numerals and natural numbers. By TRUE we denote the set of all true closed literals. Finite sets of formulas are called sequents.

We use the following syntactic variables: $s, t$ for terms, $A, B, C, D, F$ for formulas, $\Gamma, \Delta$ for sequents, $\alpha, \beta, \gamma$ for ordinals, $i, j, k, l, m, n$ for natural numbers (and numerals).

As far as sequents are concerned we usually write $A_{1}, \ldots, A_{n}$ for $\left\{A_{1}, \ldots, A_{n}\right\}$, and $A, \Gamma, \Delta$ for $\{A\} \cup \Gamma \cup \Delta$, etc.

\section{Proof systems}

A proof system $\mathfrak{S}$ is given by

- a set of formal expressions called inference symbols (syntactic variable $\mathcal{I}$ )

- for each inference symbol $\mathcal{I}$ a set $|\mathcal{I}|$, a sequent $\Delta(\mathcal{I})$ and a family of sequents $\left(\Delta_{\iota}(\mathcal{I})\right)_{\iota \in|\mathcal{I}|}$.

\section{NOTATION}

By writing $(\mathcal{I}) \frac{\ldots \Delta_{\iota} \ldots(\iota \in I)}{\Delta}$

we declare $\mathcal{I}$ as an inference symbol with $|\mathcal{I}|=I, \Delta(\mathcal{I})=\Delta, \Delta_{\iota}(\mathcal{I})=\Delta_{\iota}$.

If $|\mathcal{I}|=\{0, \ldots, n-1\}$ we write $\frac{\Delta_{0} \Delta_{1} \ldots \Delta_{n-1}}{\Delta}$ instead of $\frac{\ldots \Delta_{\iota} \ldots(\iota \in I)}{\Delta}$.

Up to a few exceptions the sequents $\Delta(\mathcal{I}), \Delta_{\iota}(\mathcal{I})$ are singletons or empty.

\section{Definition}

The figure $\frac{\ldots \Gamma_{\iota} \ldots(\iota \in I)}{\Gamma} \mathcal{I}$ is called a (correct) $\mathfrak{S}$-inference iff

$\mathcal{I} \in \mathfrak{S}$ and $|\mathcal{I}|=I$ and $\Delta(\mathcal{I}) \subseteq \Gamma$ and $\forall \iota \in I\left(\Gamma_{\iota} \subseteq \Gamma, \Delta_{\iota}(\mathcal{I})\right)$.

The infinitary proof system $Z^{\infty}$ ( $\omega$-arithmetic)

$\left(\mathrm{Ax}_{A}\right) \bar{A} \quad$ if $A \in \mathrm{TRUE}_{0}$.

$$
\begin{array}{ll}
\left(\bigwedge_{A_{0} \wedge A_{1}}\right) \frac{A_{0} A_{1}}{A_{0} \wedge A_{1}} & \left(\bigvee_{A_{0} \vee A_{1}}^{k}\right) \frac{A_{k}}{A_{0} \vee A_{1}}(k \in\{0,1\}) \\
\left(\bigwedge_{\forall x A}\right) \frac{\ldots A_{x}(i) \ldots(i \in \mathbb{N})}{\forall x A} & \left(\bigvee_{\exists x A}^{k}\right) \frac{A_{x}(k)}{\exists x A}(k \in \mathbb{N}) \\
\left(\mathrm{Cut}_{C}\right) \frac{C \quad \neg C}{\emptyset} & (\operatorname{Rep}) \frac{\emptyset}{\emptyset}
\end{array}
$$

Note:

To avoid a possible misunderstanding we stress that $|\operatorname{Rep}|=\{0\}$ while $\left|A x_{A}\right|=\emptyset$.

\section{Inductive definition of $\mathbf{Z}^{\infty}$-derivations}

If $\Gamma$ is a sequent, $\alpha$ an ordinal, $\mathcal{I} \in \mathbf{Z}^{\infty}$, and $\left(\mathrm{d}_{i}\right)_{i \in I}$ a family of $\mathbf{Z}^{\infty}$-derivations such that $\frac{\ldots \Gamma\left(\mathrm{d}_{i}\right) \ldots(i \in I)}{\Gamma} \mathcal{I}$ is a correct $\mathbf{Z}^{\infty}$-inference and $\forall i \in I\left(\mathrm{o}\left(\mathrm{d}_{i}\right)<\alpha\right)$ 
then the tree $\mathrm{d}:=\left\{\frac{\ldots \mathrm{d}_{i} \ldots(i \in I)}{\mathcal{I}: \Gamma: \alpha}\right.$ is a $\mathbf{Z}^{\infty}$-derivation with

$\Gamma(\mathrm{d}):=\Gamma, \operatorname{last}(\mathrm{d}):=\mathcal{I}, \mathrm{o}(\mathrm{d}):=\alpha, \mathrm{d}(i):=\mathrm{d}_{i}$

and $\operatorname{deg}(\mathrm{d}):= \begin{cases}\max \left\{\operatorname{rk}(C)+1, \operatorname{deg}\left(\mathrm{d}_{0}\right), \operatorname{deg}\left(\mathrm{d}_{1}\right)\right\} & \text { if } \mathcal{I}=\text { Cut }_{C} \\ \sup \left\{\operatorname{deg}\left(\mathrm{d}_{i}\right): i \in I\right\} & \text { otherwise }\end{cases}$

$\Gamma(\mathrm{d})$ is called the endsequent of $\mathrm{d}, \mathrm{o}(\mathrm{d})$ the ordinal of d, last(d) the last inference (symbol) of $\mathrm{d}$, and $\mathrm{d}(i)$ the $i$-th immediate subderivation of $\mathrm{d}$.

We use $d, d_{0}, \ldots$ as syntactic variables for $\mathbf{Z}^{\infty}$-derivations.

Abbreviation $\mathrm{d} \vdash_{m}^{\alpha} \Gamma: \Longleftrightarrow \Gamma(\mathrm{d}) \subseteq \Gamma \& \operatorname{deg}(\mathrm{d}) \leq m \& \mathrm{o}(\mathrm{d})=\alpha$.

\section{Cut-elimination for $\mathbf{Z}^{\infty}$}

\section{Theorem 1 and Definition}

Let $C$ be given. We define an operator $\mathcal{R}_{C}$ such that:

$\mathrm{d}_{0} \vdash_{m}^{\alpha} \Gamma, C \& \mathrm{~d}_{1} \vdash_{m}^{\beta} \Gamma, \neg C \& \operatorname{rk}(C) \leq m \Longrightarrow \mathcal{R}_{C}\left(\mathrm{~d}_{0}, \mathrm{~d}_{1}\right) \vdash_{m}^{\alpha \# \beta} \Gamma$.

Proof by induction on $\alpha \# \beta$ :

W.l.o.g. we may assume that $\Gamma=\left(\Gamma\left(\mathrm{d}_{0}\right) \backslash\{C\}\right) \cup\left(\Gamma\left(\mathrm{d}_{1}\right) \backslash\{\neg C\}\right)$.

Case 1. $C \notin \Delta(\mathcal{I})$ where $\mathcal{I}:=\operatorname{last}\left(\mathrm{d}_{0}\right)$ :

Then $\Delta(\mathcal{I}) \subseteq \Gamma$, and $\mathrm{d}_{0}(i) \vdash_{m}^{\alpha_{i}} \Gamma, C, \Delta_{i}(\mathcal{I})$ with $\alpha_{i}<\alpha$, for all $i \in|\mathcal{I}|$.

By IH we get $\mathcal{R}_{C}\left(\mathrm{~d}_{0}(i), \mathrm{d}_{1}\right) \vdash_{m}^{\alpha_{i} \# \beta} \Gamma, \Delta_{i}(\mathcal{I})$ for all $i \in|\mathcal{I}|$.

Hence $\mathcal{R}_{C}\left(\mathrm{~d}_{0}, \mathrm{~d}_{1}\right):=\left\{\frac{\ldots \mathcal{R}_{C}\left(\mathrm{~d}_{0}(i), \mathrm{d}_{1}\right) \ldots(i \in|\mathcal{I}|)}{\mathcal{I}: \Gamma: \alpha \# \beta}\right.$ is a derivation as required.

Case 1'. $\neg C \notin \Delta\left(\right.$ last $\left.\left(\mathrm{d}_{1}\right)\right)$ : symmetric to Case 1 .

Case 2. $C \in \Delta\left(\right.$ last $\left.\left(\mathrm{d}_{0}\right)\right)$ and $\neg C \in \Delta\left(\right.$ last $\left.\left(\mathrm{d}_{1}\right)\right)$ :

Then $\operatorname{rk}(C) \neq 0$, since $C$ and $\neg C$ cannot both be true literals.

Case 2.1. $C=\forall x A(x)$ : Then $\neg C=\exists x \neg A(x)$, last $\left(d_{1}\right)=\bigvee_{\neg C}^{k}$, and $\mathrm{d}_{0}(i) \vdash_{m}^{\alpha_{i}} \Gamma, C, A(i)$ with $\alpha_{i}<\alpha$, for all $i \in \mathbb{N}$,

$\mathrm{d}_{1}(0) \vdash_{m}^{\beta_{0}} \Gamma, C, \neg A(k)$ with $\beta_{0}<\beta$.

By IH we get $\mathcal{R}_{C}\left(\mathrm{~d}_{0}(k), \mathrm{d}_{1}\right) \vdash_{m}^{\alpha_{k} \# \beta} \Gamma, A(k)$ and $\mathcal{R}_{C}\left(\mathrm{~d}_{0}, \mathrm{~d}_{1}(0)\right) \vdash_{m}^{\alpha \# \beta_{0}} \Gamma, \neg A(k)$.

Further $\operatorname{rk}(A(k))<\operatorname{rk}(C) \leq m$.

Hence $\mathcal{R}_{C}\left(\mathrm{~d}_{0}, \mathrm{~d}_{1}\right):=\left\{\frac{\mathcal{R}_{C}\left(\mathrm{~d}_{0}(k), \mathrm{d}_{1}\right) \quad \mathcal{R}_{C}\left(\mathrm{~d}_{0}, \mathrm{~d}_{1}(0)\right)}{\text { Cut }_{A(k)}: \Gamma: \alpha \# \beta}\right.$.

Case 2.2.-2.4. $C=\exists x A$ or $A_{0} \wedge A_{1}$ or $A_{0} \vee A_{1}$ : analogous to Case 2.1 .

\section{Theorem 2 and Definition}

We define an operator $\mathcal{E}$ such that: $\mathrm{d} \vdash_{m+1}^{\alpha} \Gamma \Longrightarrow \mathcal{E}(\mathrm{d}) \vdash_{m}^{\omega^{\alpha}} \Gamma$.

Proof by induction on $\alpha$ :

W.l.o.g. we may assume that $\Gamma=\Gamma(\mathrm{d})$.

Case 1. last $(\mathrm{d})=\mathrm{Cut}_{C}$ :

Then $\operatorname{rk}(C) \leq m$ and $\mathrm{d}(0) \vdash_{m+1}^{\alpha_{0}} \Gamma, C, \mathrm{~d}(1) \vdash_{m+1}^{\alpha_{1}} \Gamma, \neg C$ with $\alpha_{0}, \alpha_{1}<\alpha$.

By IH we get $\mathcal{E}(\mathrm{d}(0)) \vdash_{m}^{\omega^{\alpha} 0} \Gamma, C$ and $\mathcal{E}(\mathrm{d}(1)) \vdash_{m}^{\omega^{\alpha_{1}}} \Gamma, \neg C$. 
Hence by Theorem $1 \mathcal{R}_{C}(\mathcal{E}(\mathrm{d}(0)), \mathcal{E}(\mathrm{d}(1))) \vdash_{m}^{\omega^{\alpha}} \# \omega^{\alpha}{ }^{\alpha}$, and $\mathcal{E}(\mathrm{d}):=\left\{\frac{\mathcal{R}_{C}(\mathcal{E}(\mathrm{d}(0)), \mathcal{E}(\mathrm{d}(1)))}{\operatorname{Rep}: \Gamma: \omega^{\alpha}}\right.$ is a derivation as required.

Case 2. otherwise: $\mathcal{E}(\mathrm{d}):=\left\{\frac{\ldots \mathcal{E}(\mathrm{d}(i)) \ldots(i \in|\mathcal{I}|)}{\mathcal{I}: \Gamma: \omega^{\alpha}}\right.$ where $\mathcal{I}:=\operatorname{last}(\mathrm{d})$.

Remark In the whole paper $\lambda \xi \cdot \omega^{\xi}$ could be replaced by any ordinal function $f$ such that $\forall \alpha_{0}, \alpha_{1}, \alpha\left(\alpha_{0}, \alpha_{1}<\alpha \Rightarrow f\left(\alpha_{0}\right) \# f\left(\alpha_{1}\right)<f(\alpha)\right)$.

\section{$\S 2$ The finitary system $z^{*}$}

Let $Z$ be the formal system of pure number theory (Peano arithmetic). The mathematical axioms of $Z$ are the scheme of complete induction and finitely many axioms of the shape $\forall \vec{x}\left(A_{0} \vee \ldots \vee A_{m}\right)$ where $A_{0}, \ldots, A_{m}$ are literals. In our sequent calculus the latter axioms are represented by a (prim. rec.) set $\operatorname{Ax}(Z)$ of sequents such that

(i) $\Delta \in \operatorname{Ax}(Z) \& A \in \Delta \Rightarrow A$ is a literal,

(ii) $\Delta \in \operatorname{Ax}(Z) \Rightarrow \Delta_{\vec{x}}(\vec{t}) \in \operatorname{Ax}(Z)$,

(iii) $\Delta \in \operatorname{Ax}(Z) \& \mathrm{FV}(\Delta)=\emptyset \Rightarrow \Delta \cap \operatorname{TRUE}_{0} \neq \emptyset$.

Definition of the finitary proof system $Z^{*}$

The inference symbols of $\mathbf{Z}^{*}$ are

$$
\begin{array}{ll}
\left(\mathrm{Ax}_{\Delta}\right) \frac{\pi}{\Delta} \text { if } \Delta \in \operatorname{Ax}(Z), & \left(\bigwedge_{\forall x A}^{y}\right) \frac{A_{x}(y)}{\forall x A}, \quad\left(\bigvee_{\exists x A}^{t}\right) \frac{A_{x}(t)}{\exists x A}, \\
\left(\operatorname{lnd}_{F}^{y, t}\right) \frac{\neg F, F_{y}(S y)}{\neg F_{y}(0), F_{y}(t)}, & \left(\mathrm{R}_{C}\right) \frac{C \quad \neg C}{\emptyset}, \quad(\mathrm{E}) \frac{\emptyset}{\emptyset}
\end{array}
$$

and $\bigwedge_{A_{0} \wedge A_{1}}, \bigvee_{A_{0} \vee A_{1}}^{k}$ as in $\mathbf{Z}^{\infty}$.

\section{$Z^{*}$-derivations}

$\mathbf{Z}^{*}$-derivations are defined in a somewhat different style than $\mathbf{Z}^{\infty}$-derivations. The difference is that the nodes of a $\mathbf{Z}^{*}$-derivation $h$ are labeled with inference symbols only, while the endsequent $\Gamma(h)$ and the ordinal o $(h)$ of $h$ will be computed from $h$ by structural recursion. Actually $\mathrm{Z}^{*}$-derivations will be introduced as terms (in prefix notation) built up from inference symbols $\mathcal{I}$ which we consider as $n$-ary function symbols, where $|\mathcal{I}|=\{0, \ldots, n-1\}$.

\section{Inductive Definition of $Z^{*}$-quasi-derivations}

If $\mathcal{I}$ is an $n$-ary $\mathbf{Z}^{*}$-inference symbol and $h_{0}, \ldots, h_{n-1}$ are $\mathbf{Z}^{*}$-quasi-derivations then $h:=\mathcal{I} h_{0} \ldots h_{n-1}$ is a $\mathbf{Z}^{*}$-quasi-derivation and

$$
\begin{aligned}
\Gamma(h):=\Delta(\mathcal{I}) \cup \bigcup_{i<n}\left(\Gamma\left(h_{i}\right) \backslash \Delta_{i}(\mathcal{I})\right), \\
\mathrm{o}(h):= \begin{cases}\mathrm{o}\left(h_{0}\right) \# \mathrm{o}\left(h_{1}\right) & \text { if } \mathcal{I}=\mathrm{R}_{C} \\
\mathrm{o}\left(h_{0}\right) \cdot \omega & \text { if } \mathcal{I}=\operatorname{lnd}_{F}^{y, t} \\
\omega^{\mathrm{o}\left(h_{0}\right)} & \text { if } \mathcal{I}=\mathrm{E} \\
\left.\sup _{i<n} \mathrm{o}\left(h_{i}\right)\right)+1 & \text { otherwise }\end{cases}
\end{aligned}
$$


$\operatorname{deg}(h)= \begin{cases}\max \left\{\operatorname{rk}(C), \operatorname{deg}\left(h_{0}\right), \operatorname{deg}\left(h_{1}\right)\right\} & \text { if } \mathcal{I}=\mathrm{R}_{C} \\ \max \left\{\operatorname{rk}(F), \operatorname{deg}\left(h_{0}\right)\right\} & \text { if } \mathcal{I}=\operatorname{Ind}_{F}^{y, t} \\ \operatorname{deg}\left(h_{0}\right)-1 & \text { if } \mathcal{I}=\mathrm{E} \\ \sup _{i<n} \operatorname{deg}\left(h_{i}\right) & \text { otherwise }\end{cases}$

Remark: The definitions of $\mathrm{o}(h)$ and $\operatorname{deg}(h)$ are motivated by the interpretation $h \mapsto h^{\infty}$ (introduced below) and Theorems 1,2.

\section{Inductive Definition of $Z^{*}$-derivations}

If $\mathcal{I}$ is an $n$-ary $\mathbf{Z}^{*}$-inference symbol and $h_{0}, \ldots, h_{n-1}$ are $\mathbf{Z}^{*}$-derivations then $h:=\mathcal{I} h_{0} \ldots h_{n-1}$ is a $\mathbf{Z}^{*}$-derivation if the following conditions are satisfied

- $\mathcal{I}=\bigwedge_{\forall x A}^{y} \Rightarrow y \notin \mathrm{FV}(\Gamma(h))$,

- $\mathcal{I}=\operatorname{lnd}_{F}^{y, t} \Rightarrow y \notin \mathrm{FV}(\Gamma(h))$,

- $\mathcal{I}=\bigvee_{\exists x A}^{t} \Rightarrow \mathrm{FV}(t) \subseteq \mathrm{FV}(\Gamma(h))$,

- $\mathcal{I}=\mathrm{R}_{C} \Rightarrow \mathrm{FV}(C) \subseteq \mathrm{FV}(\Gamma(h))$.

A Z $\mathbf{Z}^{*}$-derivation $h$ is called closed iff $\mathrm{FV}(\Gamma(h))=\emptyset$.

Remark: As one easily verifies the last two conditions in the above definition do not restrict the set of provable sequents. They imply the following proposition: If $h=\mathcal{I} h_{0} \ldots h_{n-1}$ is a closed $\mathbf{Z}^{*}$-derivation with $\mathcal{I} \neq \bigwedge_{\forall x A}^{y}, \operatorname{Ind}_{F}^{y, t}$ then $h_{0}, \ldots, h_{n-1}$ are closed too. If $h=\bigwedge_{\forall x A}^{y} h_{0}$ or $h=\operatorname{Ind}_{F}^{y, t} h_{0}$ is closed then $\operatorname{FV}\left(\Gamma\left(h_{0}\right)\right) \subseteq\{y\}$.

\section{Definition}

Let $\mathbf{Z}$ denote the subsystem of $\mathbf{Z}^{*}$ which arises by omitting the symbol $\mathbf{E}$. Obviously $\mathbf{Z}$ is nothing else than the Tait-style version of pure number theory $Z$.

We use $d, d_{i}\left(h, h_{i}\right)$ as syntactic variables for $\mathbf{Z}\left(\mathbf{Z}^{*}\right)$-derivations.

\section{Definition}

In the usual way we define $h(z / i)$, i.e. the result of substituting $i$ for $z$ in $h$ :

$\operatorname{Ax}_{\Delta}(z / i):=\operatorname{Ax}_{\Delta_{z}(i)}$,

$\left(\bigvee_{C}^{t} h_{0}\right)(z / i):=\bigvee_{C_{z}(i)}^{t_{z}(i)} h_{0}(z / i),\left(\bigwedge_{C} h_{0} h_{1}\right)(z / i):=\bigwedge_{C_{z}(i)} h_{0}(z / i) h_{1}(z / i)$,

$\left(\bigwedge_{C}^{z} h_{0}\right)(z / i):=\bigwedge_{C}^{z} h_{0},\left(\bigwedge_{C}^{y} h_{0}\right)(z / i):=\bigwedge_{C_{z}(i)}^{y} h_{0}(z / i)$ if $y \neq z$,

$\left(\operatorname{Ind}_{F}^{z, t} h_{0}\right)(z / i):=\operatorname{Ind}_{F}^{z, t} h_{0},\left(\operatorname{Ind}_{F}^{y, t} h_{0}\right)(z / i):=\operatorname{Ind}_{F_{z}(i)}^{y, t_{z}(i)} h_{0}(z / i)$ if $y \neq z$,

$\left(\mathrm{R}_{C} h_{0} h_{1}\right)(z / i):=\mathrm{R}_{C_{z}(i)} h_{0}(z / i) h_{1}(z / i),\left(\mathrm{E} h_{0}\right)(z / i):=\mathrm{E} h_{0}(z / i)$.

Proposition If $h$ is a $\mathbf{Z}^{*}$-derivation then also $h(z / i)$ is a $\mathbf{Z}^{*}$-derivation and $\Gamma(h(z / i)) \subseteq \Gamma(h)_{z}(i), \operatorname{deg}(h(z / i))=\operatorname{deg}(h), \mathrm{o}(h(z / i))=\mathrm{o}(h)$.

\section{Interpretation of $\mathrm{Z}^{*}$ in $\mathrm{Z}^{\infty}$}

For each closed $\mathbf{Z}^{*}$-derivation $h$ we define its interpretation $h^{\infty} \in \mathbf{Z}^{\infty}$ as follows: Let $h=\mathcal{I} h_{0} \ldots h_{n-1}, \Gamma=\Gamma(h), \alpha=\mathrm{o}(h)$ :

0. $\left(A x_{\Gamma}\right)^{\infty}:=\left\{\frac{}{A x_{A}: \Gamma: \alpha}\right.$, where $A$ is the "least" element of $\Gamma \cap \mathrm{TRUE}_{0}$,

1. $\left(\bigwedge_{\forall x A}^{y} h_{0}\right)^{\infty}:=\left\{\frac{\ldots h_{0}(y / i)^{\infty} \ldots(i \in \mathbb{N})}{\bigwedge_{\forall x A}: \Gamma: \alpha}\right.$, 
2. $\left(\mathrm{R}_{C} h_{0} h_{1}\right)^{\infty}:=\mathcal{R}_{C}\left(h_{0}^{\infty}, h_{1}^{\infty}\right)$,

3. $\left(\mathrm{E} h_{0}\right)^{\infty}:=\mathcal{E}\left(h_{0}^{\infty}\right)$,

4. $\left(\operatorname{Ind}_{F}^{y, n} h_{0}\right)^{\infty}:=\left\{\frac{\mathrm{e}_{n}}{\operatorname{Rep}: \Gamma: \alpha}\right.$ with

$\mathrm{e}_{1}:=h_{0}(y / 0)^{\infty}, \mathrm{e}_{i+1}:=\mathcal{R}_{F(i)}\left(\mathrm{e}_{i}, h_{0}(y / i)^{\infty}\right)$ for $i>0$, and $\mathrm{e}_{0}$ is the canonical

$\mathrm{Z}^{\infty}$-derivation with $\Gamma\left(\mathrm{e}_{0}\right)=\{\neg F(0), F(0)\}, \operatorname{deg}\left(\mathrm{e}_{0}\right)=0, \mathrm{o}\left(\mathrm{e}_{0}\right)=2 \mathrm{rk}(F)$.

5. Otherwise: $\left(\mathcal{I} h_{0} \ldots h_{n-1}\right)^{\infty}:=\left\{\frac{h_{0}^{\infty} \ldots h_{n-1}^{\infty}}{\mathcal{I}: \Gamma: \alpha}\right.$

Remark With the help of Theorems 1,2 one easily verifies that $h^{\infty}$ is a $\mathbf{Z}^{\infty}$ derivation with $h^{\infty} \vdash_{\operatorname{deg}(h)}^{\mathrm{o}(h)} \Gamma(h)$.

Definition of $\operatorname{tp}(h)$ and $h[i]$ for closed $\mathrm{Z}^{*}$-derivations $h$ and $i \in|\operatorname{tp}(h)|$ By (prim.) recursion on the build-up of $h$ we define an inference symbol $\operatorname{tp}(h) \epsilon$ $\mathbf{Z}^{\infty}$ and closed $\mathbf{Z}^{*}$-derivation(s) $h[i]$ in such a way that $\operatorname{tp}(h)=\operatorname{last}\left(h^{\infty}\right)$ and $(h[i])^{\infty}=h^{\infty}(i)$. The definition clauses for $h=\mathrm{R}_{C} h_{0} h_{1}$ and $h=\mathrm{E} h_{0}$ can be read off from the corresponding clauses in the definitions of $\mathcal{R}_{C}$ and $\mathcal{E}$.

1.1. $h=\operatorname{Ax}_{\Delta}: \operatorname{tp}\left(\mathrm{Ax}_{\Delta}\right):=\mathrm{Ax}_{A}$ where $A$ is the "least" element of $\Delta \cap \operatorname{TRUE}_{0}$.

1.2. $h=\bigwedge_{C} h_{0} h_{1}: \operatorname{tp}(h):=\bigwedge_{C}, h[i]:=h_{i}$.

1.3. $h=\bigwedge_{C}^{y} h_{0}: \operatorname{tp}(h):=\bigwedge_{C}, h[i]:=h_{0}(y / i)$.

1.4. $h=\bigvee_{C}^{k} h_{0}: \operatorname{tp}(h):=\bigvee_{C}^{k}, h[0]:=h_{0}$.

2. $h=\operatorname{Ind}_{F}^{y, n} h_{0}: \operatorname{tp}(h):=\operatorname{Rep}, h[0]:=e_{n}$ with

$e_{1}:=h_{0}(y / 0), e_{i+1}:=\mathrm{R}_{F(i)} e_{i} h_{0}(y / i)$ for $i>0$, and $e_{0}$ is the canonical

Z-derivation with $\Gamma\left(e_{0}\right)=\{\neg F(0), F(0)\}, \operatorname{deg}\left(e_{0}\right)=0, o\left(e_{0}\right)=1+2 \operatorname{rk}(F)$.

3. $h=\mathrm{E} h_{0}$ :

3.1. $\operatorname{tp}\left(h_{0}\right)=\mathrm{Cut}_{C}: \operatorname{tp}(h):=\operatorname{Rep}, h[0]:=\mathrm{R}_{C} \mathrm{E} h_{0}[0] \mathrm{E} h_{0}[1]$,

3.2. otherwise: $\operatorname{tp}(h):=\operatorname{tp}\left(h_{0}\right), h[i]:=\mathrm{E} h_{0}[i]$.

4. $h=\mathrm{R}_{C} h_{0} h_{1}$ :

4.1. $C \notin \Delta\left(\operatorname{tp}\left(h_{0}\right)\right): \operatorname{tp}(h):=\operatorname{tp}\left(h_{0}\right), h[i]:=\mathrm{R}_{C} h_{0}[i] h_{1}$.

4.2. $\neg C \notin \Delta\left(\operatorname{tp}\left(h_{1}\right)\right): \operatorname{tp}(h):=\operatorname{tp}\left(h_{1}\right), h[i]:=\mathrm{R}_{C} h_{0} h_{1}[i]$.

4.3. $C \in \Delta\left(\operatorname{tp}\left(h_{0}\right)\right)$ and $\neg C \in \Delta\left(\operatorname{tp}\left(h_{1}\right)\right)$ :

Then $\operatorname{rk}(C) \neq 0$, since $C$ and $\neg C$ cannot both be true literals.

4.3.1. $C=\forall x A$ : Then $\operatorname{tp}\left(h_{1}\right)=\bigvee_{\neg C}^{k}$ for some $k \in \mathbb{N}$. $\operatorname{tp}(h):=\mathrm{Cut}_{A_{x}(k)}, h[0]:=\mathrm{R}_{C} h_{0}[k] h_{1}, h[1]:=\mathrm{R}_{C} h_{0} h_{1}[0]$.

4.3.2. $C=\exists x A$ or $A_{0} \wedge A_{1}$ or $A_{0} \vee A_{1}$ : analogous to 4.3.1.

\section{Theorem 3}

For each closed $\mathbf{Z}^{*}$-derivation $h$ the following holds:

a) $\frac{\ldots \Gamma(h[i]) \ldots(i \in|\operatorname{tp}(h)|)}{\Gamma(h)} \operatorname{tp}(h)$ is a correct $\mathbf{Z}^{\infty}$-inference,

b) $\operatorname{tp}(h)=\mathrm{Cut}_{C} \Rightarrow \operatorname{rk}(C)<\operatorname{deg}(h)$, 
c) $\operatorname{deg}(h[i]) \leq \operatorname{deg}(h)$ for all $i \in|\operatorname{tp}(h)|$,

d) $\mathrm{o}(h[i])<\mathrm{o}(h)$ for all $i \in|\operatorname{tp}(h)|$.

Proof by straightforward induction on the build-up of $h$ :

We only consider two cases.

Abbreviation: $h \vdash_{m}^{\alpha} \Gamma: \Leftrightarrow \Gamma(h) \subseteq \Gamma \& \operatorname{deg}(h) \leq m \& \mathrm{o}(h)=\alpha$.

1. $h=\mathrm{R}_{C} h_{0} h_{1}$ with $C=\forall x A, \operatorname{tp}\left(h_{0}\right)=\bigwedge_{C}, \operatorname{tp}\left(h_{1}\right)=\bigvee_{\neg C}^{k}, \operatorname{tp}(h)=\mathrm{Cut}_{A(k)}$ :

Let $\Gamma:=\Gamma(h), \alpha:=\mathrm{o}\left(h_{0}\right), \beta:=\mathrm{o}\left(h_{1}\right)$, and $m:=\operatorname{deg}(h)$.

Then $h_{0} \vdash_{m}^{\alpha} \Gamma, C$ and $h_{1} \vdash_{m}^{\beta} \Gamma, \neg C$ and $\operatorname{rk}(A(k))<\operatorname{rk}(C) \leq \operatorname{deg}(h)$.

By IH we obtain $h_{0}[k] \vdash_{m}^{\alpha_{k}} \Gamma, C, A(k)$ with $\alpha_{k}<\alpha$,

and $h_{1}[0] \vdash^{\beta_{0}} \Gamma, \neg C, \neg A(k)$ with $\beta_{0}<\beta$.

Hence $h[0]=\mathrm{R}_{C} h_{0}[k] h_{1} \vdash_{m}^{\alpha_{k} \# \beta} \Gamma, A(k)$ and $h[1]=\mathrm{R}_{C} h_{0} h_{1}[0] \vdash_{m}^{\alpha \# \beta_{0}} \Gamma, \neg A(k)$

with $\alpha_{k} \# \beta, \alpha \# \beta_{0}<\alpha \# \beta=\mathrm{o}(h)$.

2. $h=\mathrm{E} h_{0}$ with $\operatorname{tp}\left(h_{0}\right)=\mathrm{Cut}_{C}$ : Then $\operatorname{tp}(h)=\operatorname{Rep}$ and $h[0]=\mathrm{R}_{C} \mathrm{E} h_{0}[0] \mathrm{E} h_{0}[1]$.

Let $\Gamma:=\Gamma\left(h_{0}\right)=\Gamma(h), \alpha:=\mathrm{o}\left(h_{0}\right)$ and $m:=\operatorname{deg}\left(h_{0}\right)-1=\operatorname{deg}(h)$.

By IH we have $\operatorname{rk}(C)<\operatorname{deg}\left(h_{0}\right) \leq m+1$ and $h_{0}[0] \vdash_{m+1}^{\alpha_{0}} \Gamma, C, h_{0}[1] \vdash_{m+1}^{\alpha_{1}} \Gamma, \neg C$ with $\alpha_{0}, \alpha_{1}<\alpha$. Hence $\mathrm{E} h_{0}[0] \vdash{ }_{m}^{\omega^{\alpha}} \Gamma, C$ and $\mathrm{E} h_{0}[1] \vdash{ }_{m}^{\omega^{\alpha_{1}}} \Gamma, \neg C$.

From this together with $\operatorname{rk}(C) \leq m$ we get $h[0]=\mathrm{R}_{C} \mathrm{E} h_{0}[0] \mathrm{E} h_{0}[1] \vdash_{m}^{\omega^{\alpha}} \# \omega^{\alpha_{1}} \Gamma$ and $\omega^{\alpha_{0}} \# \omega^{\alpha_{1}}<\omega^{\alpha}=\mathrm{o}(h)$.

\section{Corollary}

Let $\mathbf{Z}_{\perp}^{*}$ be the set of all $\mathbf{Z}^{*}$-derivations $h$ with $\Gamma(h)=\emptyset \& \operatorname{deg}(h)=0$.

a) $h \in \mathbf{Z}_{\perp}^{*} \Rightarrow h[0] \in \mathbf{Z}_{\perp}^{*} \& \mathrm{o}(h[0])<\mathrm{o}(h)$,

b) There is no Z-derivation $d$ with $\Gamma(d)=\emptyset$.

Proof:

a) $h \in \mathbf{Z}_{\perp}^{*} \stackrel{\text { Th. }}{\Rightarrow} h \in \mathbf{Z}_{\perp}^{*} \& \operatorname{tp}(d)=\operatorname{Rep} \stackrel{\text { Th. }}{\Rightarrow} h[0] \in \mathbf{Z}_{\perp}^{*} \& \mathrm{o}(h[0])<\mathrm{o}(h)$.

b) By transfinite induction up to $\varepsilon_{0}$ from a) we get $\mathbf{Z}_{\perp}^{*}=\emptyset$. Now assume that $d$ is a Z-derivation with $\Gamma(d)=\emptyset$. Let $m:=\operatorname{deg}(d)$. Then $\mathrm{E}^{m} d=\mathrm{E} \ldots \mathrm{E} d \in \mathbf{Z}_{\perp}^{*}$. Contradiction.

\section{Conclusion}

In this section we have proved the consistency of $\mathbf{Z}$ in a Gentzen style manner (i.e., by defining reduction steps on finite derivations in such a way that the assigned ordinals decrease), but we have not yet achieved a literal reconstruction of Gentzen's original consistency proof in [Ge38]. This is contained in $\S 3$.

\section{$\S 3$ Connection to Gentzen's consistency proof}

\section{Notation:}

If $d$ is a Z-derivation and $\nu$ a node (position) in $d$ then :

(i) $\left.\quad d\right|_{\nu}$ denotes the subderivation of $d$ determined by $\nu$. (Especially $\left.d\right|_{\langle\rangle}=d$.)

(ii) $h g t(d, \nu)$ is Gentzen's height (Höhe) of $\nu$ in $d$.

(iii) $O(d, \nu)$ is the ordinal which Gentzen assigns to $\nu$ in $d$.

(The definition of $h g t(d, \nu)$ and $O(d, \nu)$ can be found in the proof of Lemma 1.) 


\section{Definition}

For each $\mathbf{Z}^{*}$-derivation $h$ let $\phi(h)$ denote the $\mathbf{Z}$-derivation which results from $h$ by deleting all E's.

Definition of a $\mathbf{Z}^{*}$-derivation $\psi_{n}(d)$ for each Z-derivation $d$

1. $\psi_{n}\left(\mathrm{R}_{C} d_{0} d_{1}\right):=\mathrm{E}^{l-n} \mathrm{R}_{C} \psi_{l}\left(d_{0}\right) \psi_{l}\left(d_{1}\right)$, where $l:=\max \{n, \operatorname{rk}(C)\}$,

2. $\psi_{n}\left(\operatorname{Ind}_{F}^{y, t} d_{0}\right):=\mathrm{E}^{l-n} \operatorname{Ind}_{F}^{y, t} \psi_{l}\left(d_{0}\right)$, where $l:=\max \{n, \operatorname{rk}(F)\}$,

3. Otherwise: $\psi_{n}\left(\mathcal{I} d_{0} \ldots d_{m-1}\right):=\mathcal{I} \psi_{n}\left(d_{0}\right) \ldots \psi_{n}\left(d_{m-1}\right)$.

\section{Proposition}

$\Gamma\left(\psi_{n}(d)\right)=\Gamma(d), \operatorname{deg}\left(\psi_{n}(d)\right) \leq n, \phi\left(\psi_{n}(d)\right)=d$.

\section{Remark}

As we will see below (cf. Lemma 5$) \psi_{n}(d)$ has the following minimality property: $\forall h\left(\operatorname{deg}(h) \leq n \& \phi(h)=d \Rightarrow \mathrm{o}\left(\psi_{n}(d)\right) \leq \mathrm{o}(h)\right)$.

The rest of this section is occupied with the proof of the following Theorem.

\section{Theorem 4}

For each Z-derivation $d$ we have

a) $\mathrm{o}\left(\psi_{0}(d)\right)=O(d,\langle\rangle)$.

b) If $\Gamma(d)=\emptyset$ then $\operatorname{red}(d):=\phi\left(\psi_{0}(d)[0]\right)$ results from $d$ by a Gentzen reduction step, and $O(\operatorname{red}(d),\langle\rangle)<O(d,\langle\rangle)$.

\section{Lemma 1}

If $n=h g t(d, \nu)$ then $\mathrm{o}\left(\psi_{n}\left(\left.d\right|_{\nu}\right)\right)=O(d, \nu)$.

Proof by induction on $\left.d\right|_{\nu}$ :

1. $\left.d\right|_{\nu}=\mathrm{R}_{C} d_{0} d_{1}$ : Then $d_{i}=\left.d\right|_{\nu *\langle i\rangle}$ and $\operatorname{hgt}(d, \nu *\langle i\rangle)=l:=\max \{n, \operatorname{rk}(C)\}$. Hence by $\mathrm{IH} \mathrm{o}\left(\psi_{l}\left(d_{i}\right)\right)=O(d, \nu *\langle i\rangle)$, and thus o $\left(\psi_{n}\left(\left.d\right|_{\nu}\right)\right)=$

$\omega_{l-n}\left(\mathrm{o}\left(\psi_{l}\left(d_{0}\right)\right) \# \mathrm{o}\left(\psi_{l}\left(d_{1}\right)\right)\right)=\omega_{l-n}(O(d, \nu *\langle 0\rangle) \# O(d, \nu *\langle 1\rangle))=O(d, \nu)$.

2. $\left.d\right|_{\nu}=\operatorname{Ind}_{F}^{y, t} d_{0}$ : Then $d_{0}=\left.d\right|_{\nu *\langle 0\rangle}$ and $\operatorname{hgt}(d, \nu *\langle 0\rangle)=l:=\max \{n, \operatorname{rk}(F)\}$. Hence $\mathrm{o}\left(\psi_{n}\left(\left.d\right|_{\nu}\right)\right)=\omega_{l-n}\left(\mathrm{o}\left(\psi_{l}\left(d_{0}\right)\right) \cdot \omega\right) \stackrel{\mathrm{IH}}{=} \omega_{l-n}(O(d, \nu *\langle 0\rangle) \cdot \omega)=O(d, \nu)$.

3. $\left.d\right|_{\nu}=\mathcal{I} d_{0} \ldots d_{k-1}$ otherwise: Then $d_{i}=\left.d\right|_{\nu *\langle i\rangle}$ and $h g t(d, \nu *\langle i\rangle)=n$. Hence by IH $\mathrm{o}\left(\psi_{n}\left(d_{i}\right)\right)=O(d, \nu *\langle i\rangle)$ and thus $\mathrm{o}\left(\psi_{n}\left(\left.d\right|_{\nu}\right)\right)=\left(\sup _{i<k} \mathrm{o}\left(\psi_{n}\left(d_{i}\right)\right)\right)+1=$ $\left(\sup _{i<k} O(d, \nu *\langle i\rangle)\right)+1=O(d, \nu)$.

From Lemma 1 we get $\mathrm{o}\left(\psi_{0}\left(\left.d\right|_{\langle\rangle}\right)\right)=O(d,\langle\rangle)$, and thus Theorem 4 a is proved.

Abbreviation: $\mathrm{E}^{m} h:=\underbrace{\mathrm{E} \ldots \mathrm{E}}_{m} h$.

Definition (Nominal forms for derivations)

1. $*$ is a nominal form. Cut $(*):=\emptyset, \operatorname{hgt}^{*}(*):=0$.

2. If $\mathfrak{a}$ is a nominal form, $m \in \mathbb{N}$, and $h$ a $\mathbf{Z}^{*}$-derivation then $\mathbf{E}^{m} \mathrm{R}_{C} \mathfrak{a} h$ and $\mathrm{E}^{m} \mathrm{R}_{C} h \mathfrak{a}$ are nominal forms. $\operatorname{Cut}\left(\mathrm{E}^{m} \mathrm{R}_{C} \mathfrak{a} h\right):=\operatorname{Cut}(\mathfrak{a}) \cup\{C\}, \operatorname{Cut}\left(\mathrm{E}^{m} \mathrm{R}_{C} h \mathfrak{a}\right):=\operatorname{Cut}(\mathfrak{a}) \cup\{\neg C\}$, $\operatorname{hgt}^{*}\left(\mathrm{E}^{m} \mathrm{R}_{C} \mathfrak{a} h\right):=\operatorname{hgt}^{*}\left(\mathrm{E}^{m} \mathrm{R}_{C} h \mathfrak{a}\right):=m+\operatorname{hgt}^{*}(\mathfrak{a})$. 
We use $\mathfrak{a}, \mathfrak{b}, \mathfrak{c}$ as syntactic variables for nominal forms.

\section{Definition}

$\operatorname{hgt}(\mathfrak{a}):=\sup \{\operatorname{rk}(C): C \in \operatorname{Cut}(\mathfrak{a})\}$,

$\mathfrak{a}\{q\}:=$ the result of substituting $q$ for $*$ in $\mathfrak{a}\left(q\right.$ a nominal form or $\mathbf{Z}^{*}$-derivation).

\section{Lemma 2}

$\psi_{n}(d)=\mathfrak{a}\left\{h^{\prime}\right\} \Rightarrow n+\operatorname{hgt}^{*}(\mathfrak{a})=\max \{n, \operatorname{hgt}(\mathfrak{a})\}$.

Proof by induction on $\mathfrak{a}$ :

1. $\mathfrak{a}=*: n+\operatorname{hgt}^{*}(\mathfrak{a})=n=\max \{n, \operatorname{hgt}(\mathfrak{a})\}$.

2. $\mathfrak{a}=\mathrm{E}^{m} \mathrm{R}_{C} \mathfrak{a}_{0} h_{1}$ :

Then $d=\mathrm{R}_{C} d_{0} d_{1}$ and $\psi_{n}(d)=\mathrm{E}^{l-n} \mathrm{R}_{C} \psi_{l}\left(d_{0}\right) \psi_{l}\left(d_{1}\right)$ with $l:=\max \{\operatorname{rk}(C), n\}$. This yields $\mathrm{E}^{m} \mathrm{R}_{C} \mathfrak{a}_{0}\left\{h^{\prime}\right\} h_{1}=\mathfrak{a}\left\{h^{\prime}\right\}=\mathrm{E}^{l-n} \mathrm{R}_{C} \psi_{l}\left(d_{0}\right) \psi_{l}\left(d_{1}\right)$ and then $m=l-n$ and $\mathfrak{a}_{0}\left\{h^{\prime}\right\}=\psi_{l}\left(d_{0}\right)$. Hence $n+\operatorname{hgt}^{*}(\mathfrak{a})=l+\operatorname{hgt}^{*}\left(\mathfrak{a}_{0}\right) \stackrel{\mathrm{IH}}{=} \max \left\{l, \operatorname{hgt}\left(\mathfrak{a}_{0}\right)\right\}=$ $\max \left\{n, \operatorname{rk}(C), \operatorname{hgt}\left(\mathfrak{a}_{0}\right)\right\}=\max \{n, \operatorname{hgt}(\mathfrak{a})\}$.

\section{Corollary}

$\psi_{0}(d)=\mathfrak{a}\left\{\mathfrak{b}\left\{h^{\prime}\right\}\right\} \Rightarrow \operatorname{hgt}(\mathfrak{a}\{\mathfrak{b}\})=\operatorname{hgt}(\mathfrak{a})+\operatorname{hgt}^{*}(\mathfrak{b})$.

Proof: $\operatorname{hgt}(\mathfrak{a}\{\mathfrak{b}\}) \stackrel{\text { L.2 }}{=} \operatorname{hgt}^{*}(\mathfrak{a}\{\mathfrak{b}\})=\operatorname{hgt}^{*}(\mathfrak{a})+\operatorname{hgt}^{*}(\mathfrak{b}) \stackrel{\text { L.2 }}{=} \operatorname{hgt}(\mathfrak{a})+\operatorname{hgt}^{*}(\mathfrak{b})$

\section{Definition}

A $Z^{*}$-derivation $h$ is called regular iff for every subterm $\mathrm{E} h_{0}$ of $h$ we have last $\left(h_{0}\right) \in$ $\left\{\mathrm{E}, \mathrm{R}_{C}, \operatorname{Ind}_{F}^{y, t}\right\}$. - Obviously each $\psi_{n}(d)$ is regular.

$C[k]:= \begin{cases}A_{x}(k) & \text { if } C=Q x A \text { with } Q \in\{\forall, \exists\} \\ A_{k} & \text { if } C=A_{0} \circ A_{1} \text { with } \circ \in\{\wedge, \vee\} \text { and } k \in\{0,1\}\end{cases}$

\section{Lemma 3}

Let $h$ be a closed $\mathbf{Z}^{*}$-derivation.

a) If $\operatorname{tp}(h)=$ Rep then there are $\mathfrak{a}, h^{\prime}$ such that $h=\mathfrak{a}\left\{h^{\prime}\right\}, h[0]=\mathfrak{a}\left\{h^{\prime}[0]\right\}$ and either $h^{\prime}=\mathrm{E}^{m} \operatorname{Ind}_{F}^{y, t} h^{\prime \prime}$ or $h^{\prime}=\mathrm{E}^{m+1} h^{\prime \prime} \& \operatorname{tp}\left(h^{\prime \prime}\right)=\mathrm{Cut}_{B}$.

b) If $\operatorname{tp}(h)=\mathrm{Cut}_{B}$ then there are $\mathfrak{b}, C, h_{0}, h_{1}$ such that $\operatorname{hgt}^{*}(\mathfrak{b})=0$, $h=\mathfrak{b}\left\{\mathrm{R}_{C} h_{0} h_{1}\right\}, h[i]=\mathfrak{b}\left\{\left(\mathrm{R}_{C} h_{0} h_{1}\right)[i]\right\}$, and either

(1) $\operatorname{tp}\left(h_{0}\right)=\bigwedge_{C} \& \operatorname{tp}\left(h_{1}\right)=\bigvee_{\neg C}^{k} \& B=C[k]$ or

(2) $\operatorname{tp}\left(h_{0}\right)=\bigvee_{C}^{k} \& \operatorname{tp}\left(h_{1}\right)=\bigwedge_{\neg C} \& B=C[k]$.

c) If $h$ is regular and $\operatorname{tp}(h)=\bigwedge_{C}$ or $\bigvee_{C}^{k}$ then there are $\mathfrak{c}, h_{0}\left[, h_{1}\right]$ such that $C \notin \operatorname{Cut}(\mathfrak{c})$ and $\left[h=\mathfrak{c}\left\{\bigwedge_{C}^{y} h_{0}\right\} \& h[i]=\mathfrak{c}\left\{h_{0}(y / i)\right\}\right]$ or $\left[h=\mathfrak{c}\left\{\bigwedge_{C} h_{0} h_{1}\right\} \& h[i]=\mathfrak{c}\left\{h_{i}\right\}\right]$ or $\left[h=\mathfrak{c}\left\{\bigvee_{C}^{k} h_{0}\right\} \& h[0]=\mathfrak{c}\left\{h_{0}\right\}\right]$.

Proof:

a) By definition of $\operatorname{tp}(h)$ one of the following cases holds:

1. $h=\mathrm{E}^{m} \operatorname{Ind} d_{F}^{y, t} \tilde{h}$ : Then $\mathfrak{a}:=*, h^{\prime}:=h$.

2. $h=\mathrm{E}^{n} \tilde{h}$ with last $(\tilde{h}) \neq \mathrm{E}$, Ind: 
2.1. $\operatorname{tp}(\tilde{h})=\mathrm{Cut}_{B} \& n>0$ : Then $\mathfrak{a}:=*, h^{\prime}:=h$.

2.2. $\operatorname{tp}(\tilde{h})=\operatorname{Rep}$ : Then $\tilde{h}=\mathrm{R}_{C} h_{0} h_{1}$ and (w.l.o.g) $\operatorname{tp}\left(h_{0}\right)=$ Rep. By IH $h_{0}=$ $\mathfrak{a}_{0}\left\{h^{\prime}\right\}$ with $h_{0}[0]=\mathfrak{a}_{0}\left\{h^{\prime}[0]\right\}$ and $h^{\prime}=\mathrm{E}^{m} \operatorname{Ind}{ }_{F}^{y, t} h^{\prime \prime}$ or $h^{\prime}=\mathrm{E}^{m+1} h^{\prime \prime} \& \operatorname{tp}\left(h^{\prime \prime}\right)=$ Cut $_{B}$. Now for $\mathfrak{a}:=\mathrm{E}^{n} \mathrm{R}_{C} \mathfrak{a}_{0} h_{1}$ we have $h=\mathfrak{a}\left\{h^{\prime}\right\}$ and $h[0]=\mathrm{E}^{n} \mathrm{R}_{C} h_{0}[0] h_{1}=$ $\mathrm{E}^{n} \mathrm{R}_{C} \mathfrak{a}_{0}\left\{h^{\prime}[0]\right\} h_{1}=\mathfrak{a}\left\{h^{\prime}[0]\right\}$.

b) Assume that $\operatorname{tp}(h)=\mathrm{Cut}_{B}$. Then one of the following cases holds:

1. $h=\mathrm{R}_{C} h_{0} h_{1}$ and $\left[\left(\operatorname{tp}\left(h_{0}\right)=\bigwedge_{C} \& \operatorname{tp}\left(h_{1}\right)=\bigvee_{\neg C}^{k} \& B=C[k]\right)\right.$ or $\left(\operatorname{tp}\left(h_{0}\right)=\right.$ $\left.\left.\bigvee_{C}^{k} \& \operatorname{tp}\left(h_{1}\right)=\bigwedge_{\neg C} \& B=C[k]\right)\right]$ : The claim holds for $\mathfrak{b}:=*$.

2. $h=\mathrm{R}_{D} h_{0}^{\prime} h_{1}^{\prime}$ and (w.l.o.g.) $\operatorname{tp}\left(h_{0}^{\prime}\right)=\operatorname{tp}(h) \& h[i]=\mathrm{R}_{D} h_{0}^{\prime}[i] h_{1}^{\prime}$ :

By IH there are $\mathfrak{b}_{0}, C, h_{0}, h_{1}$ such that $\operatorname{hgt}^{*}\left(\mathfrak{b}_{0}\right)=0, h_{0}^{\prime}=\mathfrak{b}_{0}\left\{\mathrm{R}_{C} h_{0} h_{1}\right\}, h_{0}^{\prime}[i]=$ $\mathfrak{b}_{0}\left\{\left(\mathrm{R}_{C} h_{0} h_{1}\right)[i]\right\}$ and one of the subcases (1),(2) holds. Let $\mathfrak{b}:=\mathrm{R}_{D} \mathfrak{b}_{0} h_{1}^{\prime}$. Then $h=\mathfrak{b}\left\{\mathrm{R}_{C} h_{0} h_{1}\right\}, h[i]=\mathrm{R}_{D} h_{0}^{\prime}[i] h_{1}^{\prime}=\mathrm{R}_{D} \mathfrak{b}_{0}\left\{\left(\mathrm{R}_{C} h_{0} h_{1}\right)[i]\right\} h_{1}^{\prime}=\mathfrak{b}\left\{\left(\mathrm{R}_{C} h_{0} h_{1}\right)[i]\right\}$, and $\operatorname{hgt}^{*}(\mathfrak{b})=\operatorname{hgt}^{*}\left(\mathfrak{b}_{0}\right)=0$.

c) Assume that $h$ is regular, and $\operatorname{tp}(h)=\bigwedge_{C}$ with $C=\forall x A$.

Then one of the following cases holds:

1. $h=\bigwedge_{\forall x A}^{y} h_{0}$ : Then the claim holds for $\mathfrak{c}:=*$.

2. $h=\mathrm{E}^{m} \mathrm{R}_{D} h_{0}^{\prime} h_{1}^{\prime}$ with (w.l.o.g.) $\operatorname{tp}(h)=\operatorname{tp}\left(h_{0}^{\prime}\right)$ and $D \neq C$ : By IH $h_{0}^{\prime}=$ $\mathfrak{c}_{0}\left\{\bigwedge_{C}^{y} h_{0}\right\}$ and $h_{0}^{\prime}[i]=\mathfrak{c}_{0}\left\{h_{0}(y / i)\right\}$ with $C \notin \operatorname{Cut}\left(\mathfrak{c}_{0}\right)$. Let $\mathfrak{c}:=\mathrm{E}^{m} \mathrm{R}_{D} \mathfrak{c}_{0} h_{1}^{\prime}$. Then $h=\mathfrak{c}\left\{\bigwedge_{\forall x A}^{y} h_{0}\right\}, h[i]=\mathrm{E}^{m} \mathrm{R}_{D} h_{0}^{\prime}[i] h_{1}^{\prime}=\mathrm{E}^{m} \mathrm{R}_{D} \mathfrak{c}_{0}\left\{h_{0}(y / i)\right\} h_{1}^{\prime}=\mathfrak{c}\left\{h_{0}(y / i)\right\}$ and $C \notin\{D\} \cup \operatorname{Cut}\left(\mathfrak{c}_{0}\right)=\operatorname{Cut}(\mathfrak{c})$.

\section{Theorem 5}

Assume that $\Gamma(d)=\emptyset$ and let $h:=\psi_{0}(d)$.

Then $\operatorname{tp}(h)=$ Rep and one of the following two cases holds:

(I) $h=\mathfrak{a}\left\{\mathrm{E}^{m} \operatorname{Ind}_{F}^{y, t} h_{0}\right\}, h[0]=\mathfrak{a}\left\{\mathrm{E}^{m}\left(\operatorname{Ind}_{F}^{y, t} h_{0}\right)[0]\right\}$,

(II) $h=\mathfrak{a}\left\{\mathrm{E}^{m+1} \mathfrak{b}\left\{\mathrm{R}_{C} h_{0} h_{1}\right\}\right\}, h[0]=\mathfrak{a}\left\{\mathrm{E}^{m} \mathrm{R}_{C[k]} \mathrm{Eb}\left\{\mathrm{R}_{C} h_{0}^{-} h_{1}\right\} \mathrm{Eb}_{\{}\left\{\mathrm{R}_{C} h_{0} h_{1}^{-}\right\}\right\}$ and either

(1) $\operatorname{tp}\left(h_{0}\right)=\bigwedge_{C} \& \operatorname{tp}\left(h_{1}\right)=\bigvee_{\neg C}^{k} \& h_{0}^{-}=h_{0}[k] \& h_{1}^{-}=h_{1}[0]$ or

(2) $\operatorname{tp}\left(h_{0}\right)=\bigvee_{C}^{k} \& \operatorname{tp}\left(h_{1}\right)=\bigwedge_{\neg C} \& h_{0}^{-}=h_{0}[0] \& h_{1}^{-}=h_{1}[k]$.

Moreover $\operatorname{hgt}^{*}(\mathfrak{b})=0$ and $\operatorname{hgt}(\mathfrak{a})+m+1=\operatorname{rk}(\tilde{C})=\max \{\operatorname{hgt}(\mathfrak{b}), \operatorname{rk}(C)\}$

with $\tilde{C}:=\left\{\begin{array}{ll}C & \text { if } \mathfrak{b}=* \\ D & \text { if last }(\mathfrak{b})=\mathrm{R}_{D}\end{array}\right.$.

Proof:

We have $\Gamma(h)=\emptyset \& \operatorname{deg}(h)=0$ and therefore (by Theorem 3) tp $(h)=$ Rep.

Further $h$ is regular. Now let us assume that (I) does not hold.

Then according to L.3a) $h=\mathfrak{a}\left\{\mathrm{E}^{m+1} h^{\prime \prime}\right\}$ with $\operatorname{tp}\left(h^{\prime \prime}\right)=\mathrm{Cut}_{B}$ and $h[0]=\mathfrak{a}\left\{\left(\mathrm{E}^{m+1} h^{\prime \prime}\right)[0]\right\}=\mathfrak{a}\left\{\mathrm{E}^{m}\left(\mathrm{E} h^{\prime \prime}\right)[0]\right\}=\mathfrak{a}\left\{\mathrm{E}^{m} \mathrm{R}_{B} \mathrm{E} h^{\prime \prime}[0] \mathrm{E} h^{\prime \prime}[1]\right\}$.

By L.3b) we get $h^{\prime \prime}=\mathfrak{b}\left\{\mathrm{R}_{C} h_{0} h_{1}\right\}, h^{\prime \prime}[i]=\mathfrak{b}\left\{\left(\mathrm{R}_{C} h_{0} h_{1}\right)[i]\right\}$ with hgt $^{*}(\mathfrak{b})=0$, and - in subcase $(1)-\operatorname{tp}\left(h_{0}\right)=\bigwedge_{C} \& \operatorname{tp}\left(h_{1}\right)=\bigvee_{\neg C}^{k} \& B=C[k]$.

Putting things together yields 
$h[0]=\mathfrak{a}\left\{\mathrm{E}^{m} \mathrm{R}_{B} \mathrm{E} h^{\prime \prime}[0] \mathrm{E} h^{\prime \prime}[1]\right\}=\mathfrak{a}\left\{\mathrm{E}^{m} \mathrm{R}_{C[k]} \mathrm{Eb}\left\{\mathrm{R}_{C} h_{0}[k] h_{1}\right\} \mathrm{Eb}\left\{\mathrm{R}_{C} h_{0} h_{1}[0]\right\}\right\}$.

It remains to prove that $\operatorname{hgt}(\mathfrak{a})+m+1=\operatorname{rk}(\tilde{C})=\max \{\operatorname{hgt}(\mathfrak{b}), \operatorname{rk}(C)\}$.

Let $\mathfrak{b}^{\prime}:=\mathfrak{b}\left\{\mathrm{R}_{C} * h_{1}\right\}$. Then last $\left(\mathfrak{b}^{\prime}\right)=\mathrm{R}_{\tilde{C}}$ and $\max \left\{\operatorname{hgt}(\mathfrak{a}), \operatorname{hgt}\left(\mathfrak{b}^{\prime}\right)\right\}=$

$\operatorname{hgt}\left(\mathfrak{a}\left\{\mathrm{E}^{m+1} \mathfrak{b}^{\prime}\right\}\right) \stackrel{\text { Cor.L.2 }}{=} \operatorname{hgt}(\mathfrak{a})+\operatorname{hgt}^{*}\left(\mathrm{E}^{m+1} \mathfrak{b}^{\prime}\right)=\operatorname{hgt}(\mathfrak{a})+m+1$.

Hence $\operatorname{hgt}(\mathfrak{a})+m+1=\operatorname{hgt}\left(\mathfrak{b}^{\prime}\right)=\max \{\operatorname{hgt}(\mathfrak{b}), \operatorname{rk}(C)\}$.

Similarly we obtain $\operatorname{rk}(\tilde{C})=\operatorname{hgt}(\mathfrak{a})+m+1$.

Remark

With the above Theorem at hand the reader may now go through the relevant parts of [Ge38] and convince him/herself that indeed $\operatorname{red}(d):=\phi\left(\psi_{0}(d)[0]\right)$ results from $d$ by a reduction step in the sense of [Ge38]. To facilitate this task let us take a closer look at case (II)(1) with $C=\forall x A$. In doing so we use the following abbreviation: $d \approx h: \Leftrightarrow d=\phi(h)$. Then by combining Lemma $3 \mathrm{c}$ with Theorem 5 and writing derivations as trees we obtain the following presentation of $d$ and $\operatorname{red}(d)$ which (apart from weakenings, contractions and permutations) is exactly as in [Ge38] (pp. 34,35):
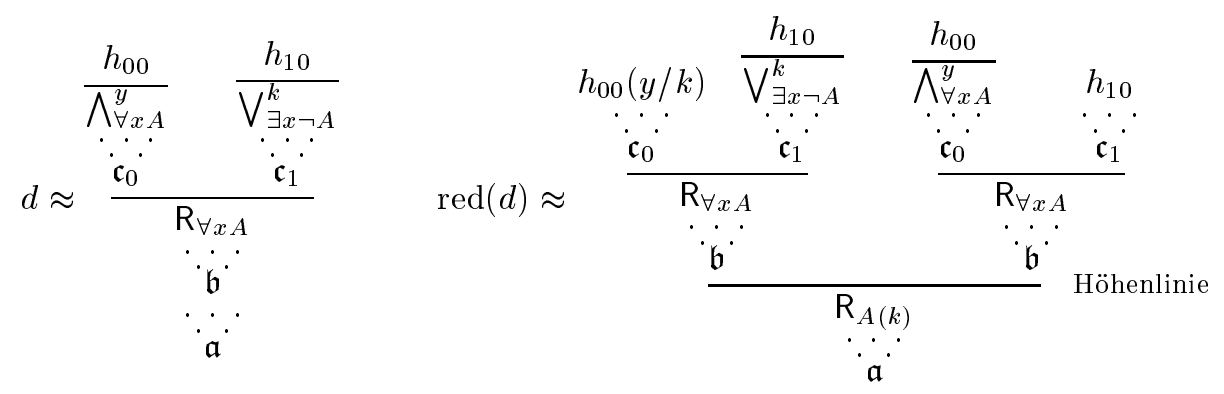

In traditional notation with sequents displayed this is:

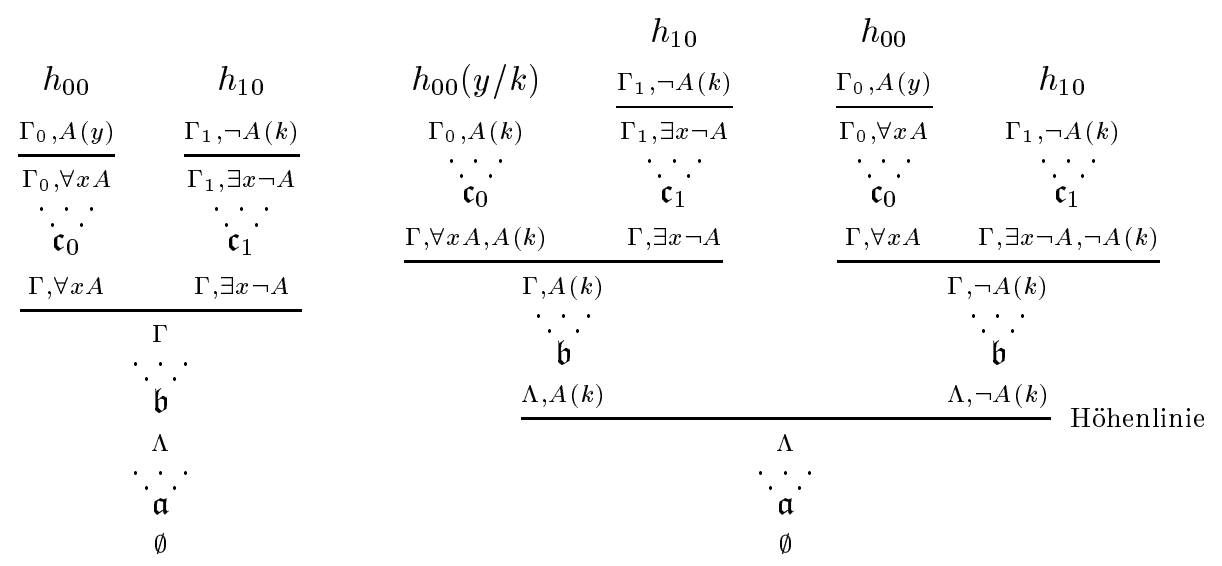

The relation $\operatorname{hgt}(\mathfrak{a})<\operatorname{rk}(\tilde{C})=\max \{\operatorname{hgt}(\mathfrak{b}), \operatorname{rk}(C)\}$ (proved above) implies that our "Höhenlinie" coincides with Gentzen's. 
Now the last part of Theorem 4, i.e. the relation $O(\operatorname{red}(d),\langle\rangle)<O(d,\langle\rangle)$, immediately follows from [Ge38]. But we think it may be useful to include an independent proof here.

Lemma $4 \quad n \leq k \Rightarrow \mathrm{o}\left(\psi_{n}(d)\right) \leq \omega_{k-n} \mathrm{o}\left(\psi_{k}(d)\right)$.

Proof:

Abbreviation: $\mathrm{o}_{n}(d):=\mathrm{o}\left(\psi_{n}(d)\right)$.

1. $d=\mathrm{R}_{C} d_{0} d_{1}$ and $l:=\max \{n, \operatorname{rk}(C)\}$ :

1.1. $l \leq k: \mathrm{o}_{n}(d)=\omega_{l-n}\left(\mathrm{o}_{l}\left(d_{0}\right) \# \mathrm{o}_{l}\left(d_{1}\right)\right) \stackrel{\mathrm{IH}}{\leq} \omega_{l-n}\left(\omega_{k-l} \mathrm{O}_{k}\left(d_{0}\right) \# \omega_{k-l} \mathrm{O}_{k}\left(d_{1}\right)\right) \leq$ $\omega_{l-n}\left(\omega_{k-l}\left(\mathrm{o}_{k}\left(d_{0}\right) \# \mathrm{o}_{k}\left(d_{1}\right)\right)\right)=\omega_{k-n} \mathrm{o}_{k}(d)$.

1.2. $n \leq k<l$ :

$\mathrm{o}_{n}(d)=\omega_{l-n}\left(\mathrm{o}_{l}\left(d_{0}\right) \# \mathrm{o}_{l}\left(d_{1}\right)\right)=\omega_{k-n} \omega_{l-k}\left(\mathrm{o}_{l}\left(d_{0}\right) \# \mathrm{o}_{l}\left(d_{1}\right)\right)=\omega_{k-n} \mathrm{o}_{k}(d)$.

2. $d=\operatorname{lnd}_{F}^{y, t}$ : analogous to 1 .

3. $d=\mathcal{I} d_{0} \ldots d_{m-1}$ otherwise: $\mathrm{o}_{n}(d)=\left(\sup _{i<m} \mathrm{o}_{n}\left(d_{i}\right)\right)+1 \stackrel{\mathrm{IH}}{\leq}$ $\left(\sup _{i<m} \omega_{k-n} \mathrm{O}_{k}\left(d_{i}\right)\right)+1 \leq \omega_{k-n}\left(\left(\sup _{i<m} \mathrm{o}_{k}\left(d_{i}\right)\right)+1\right)=\omega_{k-n}\left(\mathrm{o}_{k}(d)\right)$.

\section{Lemma 5}

$\operatorname{deg}(h) \leq n \Rightarrow \mathrm{o}\left(\psi_{n} \phi(h)\right) \leq \mathrm{o}(h)$.

Proof:

1. $h=\mathrm{E} h_{0}$ with $\operatorname{deg}\left(h_{0}\right) \leq n+1: \mathrm{o}\left(\psi_{n} \phi(h)\right)=\mathrm{o}\left(\psi_{n} \phi\left(h_{0}\right)\right) \stackrel{\text { L.4 }}{\leq}$

$\omega_{1} \mathrm{o}\left(\psi_{n+1} \phi\left(h_{0}\right)\right) \stackrel{\mathrm{IH}}{\leq} \omega_{1} \mathrm{o}\left(h_{0}\right)=\mathrm{o}(h)$.

2. $h=\mathrm{R}_{C} h_{0} h_{1}$ with $\max \left\{\operatorname{rk}(C), \operatorname{deg}\left(h_{0}\right), \operatorname{deg}\left(h_{1}\right)\right\} \leq n$ :

$\mathrm{o}\left(\psi_{n} \phi(h)\right)=\mathrm{o}\left(\psi_{n} \mathrm{R}_{C} \phi\left(h_{0}\right) \phi\left(h_{1}\right)\right) \stackrel{\mathrm{rk}(\mathrm{C}) \leq \mathrm{n}}{=} \mathrm{o}\left(\mathrm{R}_{C} \psi_{n} \phi\left(h_{0}\right) \psi_{n} \phi\left(h_{1}\right)\right)=$

$\mathrm{o}\left(\psi_{n} \phi\left(h_{0}\right)\right) \# \mathrm{o}\left(\psi_{n} \phi\left(h_{1}\right)\right) \stackrel{\mathrm{IH}}{\leq} \mathrm{o}\left(h_{0}\right) \# \mathrm{o}\left(h_{1}\right)=\mathrm{o}(h)$.

3. $h=\operatorname{Ind}_{F}^{y, t} h_{0}$ : analogous to 2 .

4. $h=\mathcal{I} h_{0} \ldots h_{m-1}$ otherwise: immediately by $\mathrm{IH}$.

Proof of $O(\operatorname{red}(d),\langle\rangle)<O(d,\langle\rangle)$ : Let $h:=\psi_{0}(d)[0]$.

From $\operatorname{deg}\left(\psi_{0}(d)\right)=0$ it follows by Theorem 3 that $\operatorname{deg}(h)=0$. Hence

$O(\operatorname{red}(d),\langle\rangle) \stackrel{\mathrm{L} .1}{=} \mathrm{o}\left(\psi_{0} \operatorname{red}(d)\right)=\mathrm{o}\left(\psi_{0} \phi(h)\right) \stackrel{\mathrm{L} .5}{\leq} \mathrm{o}(h) \stackrel{\text { Th. }}{<} \mathrm{o}\left(\psi_{0}(d)\right) \stackrel{\mathrm{L} .1}{=} O(d,\langle\rangle)$.

\section{References}

[Ar96a] Arai, T.: Consistency Proof via Pointwise Induction. Preprint (1996)

[Ar96b] Arai, T.: Proof Theory for Theories of Ordinals I: Reflecting Ordinals. Preprint (1996)

[Ar97a] Arai, T.: Proof Theory for Theories of Ordinals II: $\Sigma_{1}$-stability.

Preprint (1997) 
[Ar97b] Arai, T.: Proof Theory for Theories of Ordinals III: $\Pi_{1}$-collection. Preprint (1997)

[BS88] Buchholz, W. and Schütte, K.: Proof Theory of Impredicative Subsystems of Analysis. Studies in Proof Theory, Monographs 2. Napoli: Bibliopolis 1988

[Bu91] Buchholz, W.: Notation systems for infinitary derivations. Arch. Math. Logic 30, pp. 277-296 (1991)

[Bu97] Buchholz, W.: Explaining the Gentzen-Takeuti reduction steps. Preprint (1997)

[Ge38] Gentzen, G.: Neue Fassung des Widerspruchsfreiheitsbeweises für die reine Zahlentheorie. Forschungen zur Logik und zur Grundlegung der exakten Wissenschaften. Neue Folge 4, pp. 19-44 (1938)

[Mi75] Mints, G.: Finite Investigations of Transfinite Derivations. In: Mints,G., Selected Papers in Proof Theory. Studies in Proof Theory, Monographs 3. Napoli: Bibliopolis 1992. Russian original: Zapiski nauchnykh seminarov, LOMI 49 (1975) pp. 67-122

[Ra91] Rathjen, M.: Proof Theoretic Analysis of KPM. Arch. Math. Logic 30 (1991) pp. 377-403

[Ra94] Rathjen, M.: Proof Theory of Reflection. APAL 68 (1994) pp. 181-224

[Ra95] Rathjen, M.: Recent Advances in Ordinal Analysis: $\Pi_{2}^{1}-\mathrm{CA}$ and related systems. The Bulletin of Symbolic Logic 1/4 (1995) pp. 468-485

[Sch51] Schütte, K.: Beweistheoretische Erfassung der unendlichen Induktion in der Zahlentheorie. Math. Ann. 122, pp. 369-389 (1951)

[Ta68] Tait, W.W.: Normal Derivability in Classical Logic. In: Barwise, J. (ed.) The syntax and semantics of infinitary languages. (Lect. Notes Math., vol.72, pp. 204-236) Berlin Heidelberg New York: Springer 1968 